\title{
Male Genital Examinations: Special Considerations and Pearls for Dermatologists
}

\author{
Alexander M. Cartron, BS; John G. Zampella, MD
}

\section{PRACTICE POINTS}

- Genital examinations are an important aspect of comprehensive dermatologic care for male patients.

- Unintentional patient erections are unique to male patients and should be addressed professionally, depending on the patient's reaction.

- In addition to being mindful of body language and tone, dermatologists may consider involving a chaper one when performing genital examinations to optimize patient experience.
M en have unique dermatologic needs yet are significantly less likely than women to visit a dermatologist's office. ${ }^{1}$ Male patients might have preconceived notions about the nature of dermatology visits and necessary areas of the body to be examined: For example, male patients might associate the genital examination with a urologist and not expect a dermatologist to complete such a seemingly private examination. ${ }^{2}$

Genital examinations are currently underperformed: Only one-quarter of dermatologists report examining a male patient's genitals at most or all visits. ${ }^{3}$ In this commentary, we discuss the importance of genital examinations in men's dermatology, specific issues that can arise, and strategies to enhance the quality and frequency of genital examinations in male patients.

\section{Invaluable Aspect of Care}

Thorough inspection of a male patient's genital region is an important part of conducting a total-body skin examination (TBSE) for routine surveillance and evaluation of genital dermatoses. Sexually transmitted infections, warts, and other common lesions can be missed in diagnosis without careful inspection of the genital region. Additionally, scrotal malignancies, such as primary and metastatic melanoma and basal cell carcinoma, though rare, might be overlooked until symptoms become severe. ${ }^{4,5}$

There is no substitute for a physical examination but, in certain circumstances, it might be appropriate for a dermatologist to ask a patient if he has concerning lesions on his genitals. However, patients often are unsure of worrisome signs, and areas of the perineum might not be easily visible to a patient. Genital inspection during the physical examination allows for a teachable moment, during which the dermatologist can educate the patient about benign lesions and variants, such as pearly penile papules, seborrheic keratoses, and sebaceous cysts. ${ }^{6}$ These lesions might not require intervention but should be monitored for atypical features or infection. ${ }^{6}$

Also, the dermatologist might incidentally discover transmissible lesions, such as condylomata caused by human papillomavirus, which has been shown to be present in approximately $50 \%$ of men in the United States ${ }^{7}$ - many of whom are unaware. Inflammatory dermatoses, such as psoriasis, often affect the genitals

Mr. Cartron is from the Department of Dermatology, University of Maryland School of Medicine, Baltimore. Dr. Zampella is from the Ronald O. Perelman Department of Dermatology, New York University School of Medicine, New York.

The authors report no conflict of interest.

Correspondence: Alexander M. Cartron, BS, Department of Dermatology, University of Maryland School of Medicine, 419 W Redwood St, Ste 235, Baltimore, MD 21201 (alexander.cartron@som.umaryland.edu).

doi:10.12788/cutis.0164 
and go unnoticed; prompt intervention can decrease the likelihood of complications. ${ }^{6}$

\section{Protocol for Genital Examinations}

To examine the genitals, all surfaces of the penis, scrotum, and perineum should be evaluated, with anatomic and pathologic variants noted. The patient or physician should stretch the penis, maneuvering it in multiple directions so that all aspects can be examined. In uncircumcised men, the foreskin should be retracted so that the head of the penis can be examined, followed by replacement of the foreskin by the patient. ${ }^{8}$ The scrotum also should be examined and lifted to fully view the perineum.

Providers should not grasp the penis with the whole hand but use the thumb and first finger to hold the head of the penis to maneuver it. ${ }^{8}$ Similarly, using the back of the hand and fingers to manipulate the genitals establishes boundaries and sets a clinical tone for the examination.

Unintentional Erection-Unique to the male dermatologic examination is the unintentional patient erection; a physician might be unsure of how to approach such a potentially awkward situation. An erection is not always an indication of sexual arousal; rather, it can reflect an autonomic reflex in response to physical stimulation. Erections occur commonly in health care settings, especially if the genitals are being manipulated. ${ }^{9}$

Generally, the course of action here depends on the patient's response. ${ }^{10}$ For patients who appear unbothered, it might be appropriate to ignore the erection and proceed with the examination, especially if the physician is not actively examining the genital region. If the patient appears embarrassed, the physician can say "This is completely normal" or "Random erections are common" to normalize the situation. Joking or laughing should be avoided. For a patient who appears upset, the physician can step outside the room for a brief period to give the patient privacy, then re-enter and ask him if he is comfortable continuing with the examination.

When a patient develops an erection, the physician might become uncomfortable and, consciously or subconsciously, increase the pace of the examination, which is a natural tendency, but expediency at the expense of comprehensive care is inappropriate.

Examiner's Body Language and Tone-Throughout the genital examination, the physician should be mindful of their comments and body language to avoid exacerbating patient vulnerability. Using anatomic terms, rather than colloquial ones, to describe the genitalia is advised to prevent misunderstanding and maintain a professional clinical environment. Providers should be prepared to explain anatomic terms because some patients are not familiar with medical terminology.

Presence of a Chaperone-Involving a chaperone, as recommended by the American Medical Association, might make a patient more comfortable and alleviate potential misunderstanding. Still, physicians should be aware that some patients might feel uncomfortable with a chaperone, interpreting their presence as an expectation of impropriety. ${ }^{11}$ Universal offering of a chaperone to all patients, regardless of the gender of the physician, as well as appropriate signage in the clinical environment, normalizes chaperone invitation and use.

Other Helpful Considerations-Various strategies in the male genital examination can increase patient and physician comfort and improve care:

- The patient should be offered a gown before a TBSE or any skin examination during which the genitals will be examined.

- The patient should be allowed to keep his shorts or underwear on to avoid the feeling of being naked, which can provoke anxiety. Prior to beginning the examination, disclose that it will include "under the covered areas."

- Ask the patient for permission to conduct the examination, enumerate the steps, and provide a rationale for a genital examination. These steps help gain cooperation, alleviate anticipation, and prevent surprise.

- To increase the patient's comfort level, he can be asked whether he prefers to be examined supine or standing.

- Consider allowing the patient, himself, to expose and manipulate his genitals during the examination to increase his involvement and sense of autonomy.

- For genital examinations, patients often prefer that the examiner be a physician of the same gender. Accommodating a patient's request regarding the examiner's gender might not always be possible, but the medical practice should make an honest attempt to oblige.

Lastly, providers should be cognizant of the needs of male sexual and gender minority populations (ie, gay, bisexual, transgender/gender diverse, queer or questioning, intersex, and asexual persons). For example, transgender women might retain male anatomy or have surgical alteration of the genital region that also requires evaluation. In such patient populations, the genital examination is equally important to evaluate for dermatologic conditions that require treatment.

\section{Final Thoughts}

The male genital examination is an important component of the TBSE, as dermatologists can recognize lesions before symptoms present. Robust educational methods for trainees and practitioners in dermatology are lacking, and development of curricula might be beneficial to increase comfort in performing the genital examination. Still, use of the procedures described in this commentary can normalize the men's genital examination, optimize the physical examination, and improve men's overall dermatologic health.

\section{REFERENCES}

1. Tripathi R, Knusel KD, Ezaldein HH, et al. Association of demographic and socioeconomic characteristics with differences in use of outpatient dermatology services in the United States. JAMA Dermatol. 2018;154:1286-1291. 
2. Brezinski EA, Harskamp CT, Ledo L, et al. Public perception of dermatologists and comparison with other medical specialties: results from a national survey. J Am Acad Dermatol. 2014;71:875-881.

3. Rieder EA, Mu EW, Wang J, et al. Dermatologist practices during total body skin examinations: a survey study. J Drugs Dermatol. 2018;17:516-520.

4. Gonzalez CD, Hawkes JE, Bowles TL. Malignant melanoma scrotal metastasis: the importance of the genital examination. JAAD Case Rep. 2017;3:10-12.

5. Solimani F, Juratli $\mathrm{H}$, Hoch $\mathrm{M}$, et al. Basal cell carcinoma of the scrotum: an important but easily overlooked entity. J Eur Acad Dermatol Venereol. 2018;32:E254-E255.

6. Gabrielson AT, Le TV, Fontenot C, et al. Male genital dermatology: a primer for the sexual medicine physician. Sex Med Rev. 2019;7:71-83.
7. Han JJ, Beltran TH, Song JW, et al. Prevalence of genital human papillomavirus infection and human papillomavirus vaccination rates among US adult men: National Health and Nutrition Examination Survey (NHANES) 2013-2014. JAMA Oncol. 2017; 3:810-816

8. Albaugh JA, Kellogg-Spadt S. Genital and dermatologic examination. part II: the male patient. Urol Nurs. 2003;23:366-367.

9. Dean RC, Lue TF. Physiology of penile erection and pathophysiology of erectile dysfunction. Urol Clin North Am. 2005;32:379-395.

10. Norwick P, Weston GK, Grant-Kels JM. Erection ethics. J Am Acad Dermatol. 2019;81:1225.

11. Vogel L. Chaperones: friend or foe, and to whom? CMAJ. 2012; 184:642-643. 